\title{
Selective ordering of preoperative investigations by anesthesiologists reduces the number and cost of tests
}

\author{
La demande sélective de tests préopératoires par les anesthésiologistes réduit le nom-
} bre et le coût des tests

Barry A. Finegan FRCPC, ${ }^{*}$ Saifudin Rashiq FRCPC, ${ }^{*}$ Finlay A. McAlister FRCPC, $†$ Paul O’Connor FFARCSI*

Purpose: Preoperative investigations are frequently ordered according to care maps or protocols. We hypothesized that selective ordering of investigations by anesthesiology staff would reduce the number and cost of testing.

Methods: Prospective descriptive double cohort study carried out over 17 weeks in a tertiary care preadmission clinic. In Group I, testing followed usual practice (based on standing preoperative orders) while in Group 2 testing was initiated only on the order of an attending anesthesiologist or anesthesiology resident. Postoperative complications were categorized and confirmed by an internist blinded to group assignment. Fisher's exact test, Chisquare and Student's t test were used to compare the groups as appropriate. Statistical significance was inferred at $P<0.05$.

Results: Data were obtained from 507 patients in Group I and 43। patients in Group 2. Demographics and ASA risk score were similar in both groups. The mean number of tests ordered did not differ between groups. The mean cost of investigations was reduced from $\$ 124$ in Group I to \$95 in Group $2(P<0.05)$. If data for patients assessed by staff anesthesiologists only were considered, the mean cost of testing was reduced to $\$ 73$. The number and cost of tests ordered by anesthesia residents were similar to that in Group I. More complications were noted in Group 2, but these did not appear to be related to the altered test ordering practice.

Conclusion: Selective test ordering by staff anesthesiologists reduces the number and cost of preoperative investigations. Educational efforts should be directed towards improving resident and staff preoperative test ordering practices.
Objectif : Les tests préopératoires sont souvent demandés pour satisfaire à des plans thérapeutiques ou à des protocoles. Nous avons émis l'hypothèse qu'une demande sélective de tests par les anesthésiologistes réduirait le nombre et le coût de ces tests.

Méthode : Une étude prospective et descriptive de deux cohortes a été menée pendant 17 semaines dans une clinique de soins tertiaires. Dans le groupe I, le protocole habituel a été suivi (fondé sur les règlements) tandis que dans le groupe 2, les tests ont été faits seulement sur ordre d'un anesthésiologiste traitant ou d'un résident en anesthésiologie. Les complications postopératoires ont été catégorisées et confirmées par un interniste impartial. Le test exact de Fisher, les tests du $x^{2}$ et $t$ de Student ont servi à comparer les groupes. Une signification statistique était considérée pour $P<0,05$.

Résultats : Les données ont été obtenues de 507 patients dans le groupe I et de 43 I dans le groupe 2 . Les données démographiques et la cotation des risques selon l'ASA, de même que le nombre de tests demandés étaient similaires dans les deux groupes. Le coût moyen des tests a été réduit de 124 \$ dans le groupe 1 à 95 \$ dans le groupe 2 $(P<0,05)$. En ne tenant compte que des données évaluées par les anesthésiologistes traitants, le coût moyen était réduit à 73 \$. Le nombre et le coût des tests demandés par les résidents étaient similaires à ceux du groupe 1. II y a eu plus de complications dans le groupe 2, mais elles ne semblaient pas reliées à une modification de la demande de tests.

Conclusion : La demande sélective de tests par les anesthésiologistes traitants réduit le nombre et le coût des tests préopératoires. II faudrait accentuer les efforts de formation visant à améliorer la pratique de demande de tests préopératoires par les résidents et le personnel traitant.

From the Department of Anesthesiology and Pain Medicine, ${ }^{*}$ and the Division of General Internal Medicine, Department of Medicine, $\dagger$ University of Alberta, Edmonton, Alberta, Canada.

Address correspondence to: Dr. B. A. Finegan, Department of Anesthesiology and Pain Medicine, 8-120 Clinical Sciences Building, Edmonton, Alberta T6G 2G3, Canada. Phone: 780-407-8887; Fax: 780-407-3200; E-mail: christy.hagel@ualberta.ca Attribution: Department of Anesthesiology and Pain Medicine, University of Alberta, Edmonton, Alberta, Canada.

Supported by: Departmental funding.

Accepted for publication June 3, 2004.

Revision accepted February 17, 2005. 
$\mathrm{T}$

$\mathrm{HE}$ routine ordering of screening laboratory and radiological investigations prior to surgery has been the subject of considerable criticism, resulting in the development of guidelines, ${ }^{1-3}$ computer programs ${ }^{4}$ and procedure/disease based algorithms to guide test selection. ${ }^{5,6}$ The latter are usually institution or region specific and are incorporated into clinical pathways or care maps designed to standardize pre- and postoperative management of elective surgical patients. ${ }^{6}$ Clinical pathways have proven to be cost effective, reducing laboratory and diagnostic charges without adversely affecting outcome. ${ }^{7}$ In many situations, particularly where the patient is healthy or the procedure is relatively non-invasive, no preoperative investigations are performed. ${ }^{8}$

In parallel with these developments, surgical preoperative assessment clinics have been established which obviate the need for hospital admission prior to the day of surgery. ${ }^{9}$ This process has proven to be cost-effective and well accepted by patients. ${ }^{10}$ Preadmission clinics are frequently not staffed by physicians but by nurses who function in a screening role, detecting medical or physical conditions that trigger an anesthesiology or internal medicine consultation. ${ }^{11,12}$ Indeed, even in clinics where patients are assessed preoperatively by an anesthesiologist, such as is the case in our clinic, preoperative test orders may not be individualized but rather ordered by the surgical service based on the clinical pathway for the proposed surgical procedure.

We hypothesized that a reduction in the number of diagnostic tests ordered and consequently the cost of such testing would occur if the anesthesiologist attending in the clinic assumed the responsibility for ordering them. Given the widely acknowledged influence of experience in determining what investigations might be appropriate, we also sought to find out if preoperative test ordering practices differed between resident and attending anesthesiologist staff.

\section{Methods}

This study was approved by the institutional health research Ethics Review Board and conducted at the preadmission clinic of a university tertiary referral centre. Written informed consent was obtained from the medical staff participating in the study. During the study period, patient assessment was performed by one of 34 staff anesthesiologists and six anesthesiology residents who attended the clinic daily on a rotational basis. All patients attending the clinic who were admitted to hospital following their procedure, (including those referred subsequently for subspecialty consultation by internal medicine and cardiology), apart from those scheduled for cardiac surgery or undergoing dialysis at the time of the clinic visit, were enrolled prospectively in the study.

Six months prior to the commencement of the study, input was sought from staff on what they considered core screening investigations. Pre-printed forms were developed listing these investigations. The forms allowed single tests to be selected (for example hemoglobin as distinct from complete blood count). Tests ordered outside this core group were not subject to analysis in this study.

The study was conducted in two phases. During the initial phase (Group 1), preoperative investigations were ordered as per the usual practice from preprinted orders specified in surgery-specific clinical pathways developed by surgeons, anesthesiologists and internists from our institution within the previous five years. The orders were signed by surgical rather that anesthesiology staff. Following an interval, during which the surgical, anesthesiology and nursing staff were subject to education on the study procedures, the second phase (Group 2) was initiated. In Group 2 test selection was individualized for each patient by either a staff anesthesiologist or an anesthesiology resident. In both groups, data were collected prospectively and entered into a database by a non-physician research assistant not involved in test selection or review. Patient demographics and the number of tests ordered were recorded. The total cost of each blood test, chest $x$-ray (CXR) and electrocardiogram (ECG) was obtained from our laboratory, radiology and cardiology services, respectively. These data included the professional fee component.

Following the anticipated discharge of each subject from hospital, the in-hospital medical record was reviewed by a clinical research assistant experienced in clinical data collection and blinded to group assignment, to determine the occurrence of the following perioperative complications: unplanned admission to an intensive care unit immediately following surgery, myocardial infarction, congestive heart failure, cerebrovascular accident, deep venous thrombosis, pulmonary embolism, pneumonia, renal failure or death. The occurrence of each individual complication was recorded (for example death arising in a patient that developed renal failure would give rise to two recorded complications). The record of any patient in which the aforementioned complications were noted was reviewed in a blinded fashion by an internist (F.McA.). The review assessed if the discharge diagnosis was supported by the data on file, using pre-standardized definitions for each outcome and a judgement made if the outcome could have been influenced by additional pre- 
TABLE I Demographic, ASA risk classification and surgery type of Group 1 and Group 2 patients

\begin{tabular}{|c|c|c|c|c|}
\hline & Group 1 & $\begin{array}{l}\text { Group } 2 \\
\text { All }\end{array}$ & $\begin{array}{l}\text { Group } 2 \\
\text { Staff }\end{array}$ & $\begin{array}{l}\text { Group } 2 \\
\text { Residents }\end{array}$ \\
\hline$n$ & 507 & 431 & 176 & 255 \\
\hline Age $($ mean $\pm S D)$ & $57 \pm 16$ & $58 \pm 16$ & $57 \pm 17$ & $58 \pm 16$ \\
\hline Male & $237(47 \%)$ & $211(49 \%)$ & $92(53 \%)$ & $119(47 \%)$ \\
\hline \multicolumn{5}{|l|}{ ASA classification } \\
\hline 1 & $64(13 \%)$ & 49 (11\%) & $23(13 \%)$ & $26(10 \%)$ \\
\hline 2 & $252(50 \%)$ & $220(51 \%)$ & $89(50 \%)$ & $131(51 \%)$ \\
\hline 3 & $162(32 \%)$ & $131(30 \%)$ & $54(31 \%)$ & $77(30 \%)$ \\
\hline 4 & $5(1 \%)$ & $13(3 \%)$ & $4(2 \%)$ & $9(4 \%)$ \\
\hline Data N/A & $24(5 \%)$ & $18(4 \%)$ & $6(3 \%)$ & $12(5 \%)$ \\
\hline \multicolumn{5}{|l|}{ Surgery type } \\
\hline Orthopedics & $174(34 \%)$ & $151(35 \%)$ & $68(39 \%)$ & $83(33 \%)$ \\
\hline General surgery & $173(34 \%)$ & $107(25 \%)$ & $36(20 \%)$ & $71(28 \%)$ \\
\hline Neurosurgery & $60(12 \%)$ & $60(14 \%)$ & $34(19 \%)$ & $26(10 \%)$ \\
\hline Urology & $42(8 \%)$ & $44(10 \%)$ & $13(7 \%)$ & $31(12 \%)$ \\
\hline Other & $58(11 \%)$ & $69(16 \%)$ & $25(14 \%)$ & $44(17 \%)$ \\
\hline
\end{tabular}

$\mathrm{N} / \mathrm{A}=$ not available.

operative investigations. Follow-up was limited to the duration of hospitalization after the elective surgical procedure which initiated the preadmission clinic visit.

Ordering frequency for each test and the complication rates between the groups were compared by Chisquare analysis and Fischer's exact test as appropriate. The number and cost of tests ordered in each phase was compared by Student's $t$ test. Statistical significance was inferred at $P<0.05$. The study had $100 \%$ power to detect the difference found in total test cost between the groups with an error rate of 0.001 and had $86 \%$ power to capture the recorded aggregate difference in complication rates between the groups with an error rate of 0.05 .

\section{Results}

Test ordering data were obtained from 938 subjects, 507 (54\%) in Group 1 and 431 in Group 2. The characteristics of the subjects in each group were comparable in terms of age, gender, co-morbidity (as suggested by ASA classification), as were types of scheduled surgery (Table I).

The mean number of tests ordered did not differ between the groups with complete blood count, electrolytes, creatinine and ECG being the most frequently ordered investigations (Table II). In Group 2, testing was more targeted in nature (hemoglobin rather than a complete blood count; type and screen rather than a group and cross match). CXR investigations were decreased by $50 \%$ in Group 2 . The mean cost of preoperative testing was reduced from $\$ 124$ in Group 1 to $\$ 95$ in Group $2(P<0.05)$. Anesthesiology residents ordered more tests than their staff counterparts, but the costs incurred by resident staff were still less than that calculated in Group 1 (Table II; $P<0.05$ ).

Outcome data were not available in 31 patients: five subsequently refused surgery following their preoperative visit, seven deferred surgery because of personal or family illness, ten procedures were cancelled by the surgeon and nine subjects were lost to follow-up as their surgery was performed at another institution.

There were 20 a priori defined perioperative complications in 12 patients. Four patients in Group 1 suffered four complications and eight patients in Group 2 suffered 16 complications $(P<0.05)$. There were no deaths in Group 1 but four deaths in Group $2(P<$ $0.05)$. The deaths were as follows: A 58 -yr-old female died on day 14 following an esophagogastrectomy, (she had previously received a double lung transplant). A 53-yr-old male who underwent resection for metastases in the liver died of hepatic failure. A 79-yr-old female who also underwent resection of liver metastases died from septic shock, and an 80-yr-old man with a primary diagnosis of adenocarcinoma of the colon died from septic shock subsequent to a colostomy procedure. Three patients were admitted on a non-scheduled basis to the intensive care unit. Overall complications included three episodes of heart failure, two myocardial infarctions, one stroke, one episode of pneumonia, and two occurrences of deep venous thromboses. There were four occurrences of postoperative renal failure, all of which arose in Group 2 patients. The internist reviewed the complications, he concluded in all cases that the selection of preopera- 
TABLE II Number, cost and details of tests ordered in Group 1 and Group 2

\begin{tabular}{|c|c|c|c|c|c|c|c|c|c|}
\hline & \multicolumn{2}{|c|}{ Group 1} & \multicolumn{2}{|c|}{$\begin{array}{l}\text { Group } 2 \\
\text { All }\end{array}$} & \multicolumn{2}{|c|}{$\begin{array}{l}\text { Group } 2 \\
\text { Staff }\end{array}$} & \multicolumn{3}{|c|}{$\begin{array}{l}\text { Group } 2 \\
\text { Residents }\end{array}$} \\
\hline$n$ & \multicolumn{2}{|l|}{507} & \multicolumn{2}{|l|}{431} & \multicolumn{2}{|l|}{176} & \multicolumn{3}{|l|}{255} \\
\hline $\begin{array}{l}\text { Number of tests } \\
(\text { mean } \pm \text { SD })\end{array}$ & \multicolumn{2}{|c|}{$5.6 \pm 3.5^{*}$} & \multicolumn{2}{|c|}{$5.4 \pm 3.2$} & \multicolumn{2}{|c|}{$4.3 \pm 3.2$ * $\mathrm{I}$} & \multicolumn{3}{|c|}{$6.1 \pm 3.09$} \\
\hline $\begin{array}{l}\text { Test cost } \$ C D N \\
(\text { mean } \pm S D)\end{array}$ & \multicolumn{2}{|c|}{$124 \pm 84^{*} \dagger$} & \multicolumn{2}{|c|}{$95 \pm 75^{*}$} & \multicolumn{2}{|c|}{$74 \pm 699 \mathrm{~g} \dagger$} & \multicolumn{3}{|c|}{$110 \pm 769 \dagger$} \\
\hline Individual tests & $n$ & $\%$ & $n$ & $\%$ & $n$ & $\%$ & $n$ & $\%$ & $\operatorname{Cost}(\$)$ \\
\hline CBC & 407 & $80 *+\Phi$ & 221 & $51^{*}$ & 69 & $39 \dagger$ & 152 & 609 & 15.05 \\
\hline ECG & 330 & 659 & 290 & 67 & 106 & 60 & 184 & $72 \mathbb{I}$ & 26.55 \\
\hline Electrolytes & 320 & $63+9$ & 278 & 64 & 80 & $45 \dagger$ & 198 & 78 & 13.63 \\
\hline CXR & 266 & $52^{*}+\Phi$ & 111 & $26^{*}$ & 33 & $19 \dagger$ & 78 & 319 & 110.00 \\
\hline Creatinine & 258 & $51+\pi$ & 245 & 56 & 73 & $42 \dagger$ & 172 & $68 \mathrm{I}$ & 3.44 \\
\hline Urea & 232 & $46 \dagger$ & 172 & 40 & 44 & $25 \dagger$ & 128 & 50 & 3.45 \\
\hline PT-INR & 202 & 39†ा & 164 & 38 & 42 & $24 \dagger$ & 122 & 499 & 8.00 \\
\hline PTT & 196 & $39 * \dagger$ & 118 & $27^{*}$ & 34 & $19+$ & 84 & 33 & 10.00 \\
\hline Glucose & 160 & $32 * \dagger$ & 110 & $26^{*}$ & 36 & $20 \dagger$ & 74 & 29 & 3.28 \\
\hline Type and screen & 128 & $25^{*}+9$ & 186 & $43^{*}$ & 63 & $36 \dagger$ & 123 & $48 \mathrm{I}$ & 20.00 \\
\hline Cross match & 111 & $22 * \mathbb{I}$ & 66 & $15^{*}$ & 27 & 15 & 39 & $15 \mathrm{I}$ & 30.50 \\
\hline ALP & 55 & 11 & 33 & 8 & 14 & 8 & 19 & 7 & 3.45 \\
\hline Urinalysis & 54 & 11 & 32 & 7 & 10 & 6 & 22 & 9 & 9.04 \\
\hline \multirow[t]{2}{*}{ Bilirubin } & 36 & 7 & 27 & 6 & 9 & 5 & 18 & 7 & 3.48 \\
\hline & \multicolumn{2}{|c|}{$\begin{array}{l}\text { Group } 1 \\
(\$)\end{array}$} & \multicolumn{2}{|c|}{$\begin{array}{l}\text { Group } 2 \\
\text { All }\end{array}$} & \multicolumn{2}{|c|}{$\begin{array}{l}\text { Group } 2 \\
\text { Staff }\end{array}$} & \multicolumn{2}{|c|}{$\begin{array}{l}\text { Group } 2 \\
\text { Residents }\end{array}$} & Cost \\
\hline Individual tests & $n$ & $\%$ & $n$ & $\%$ & $n$ & $\%$ & $n$ & $\%$ & \\
\hline LF studies & 18 & $4^{*}+1$ & $35^{*}$ & 8 & 6 & 3 & 29 & 119 & 48.60 \\
\hline $\mathrm{ABG}$ & 18 & 4 & 23 & 5 & 6 & 3 & 17 & 7 & 43.56 \\
\hline $\mathrm{Ca}^{2+}$ & 14 & 3 & 14 & 3 & 7 & 4 & 7 & 3 & 9.36 \\
\hline Albumin & 8 & 2 & 7 & 2 & 5 & 3 & 2 & 1 & 3.62 \\
\hline $\mathrm{Mg}^{2+}$ & 10 & 2 & 10 & 2 & 6 & 3 & 4 & 2 & 3.53 \\
\hline PO4- & 9 & 2 & 8 & 2 & 5 & 3 & 3 & 1 & 3.48 \\
\hline Hemoglobin & 5 & $1 *+\Phi$ & 151 & $35^{*}$ & 72 & $41 \dagger$ & 79 & 319 & 3.88 \\
\hline $\mathrm{K}+$ & 1 & $0.2^{*} \dagger$ & 9 & $2^{*}$ & 6 & $3 \dagger$ & 3 & 1 & 3.52 \\
\hline
\end{tabular}

* I† Data points sharing the same symbol across rows are statistically different $(P<0.05)$.

$\mathrm{CBC}=$ complete blood count; ECG = electrocardiogram; $\mathrm{CXR}=$ chest $\mathrm{x}$-ray; PT-INR = prothrombin time international normalized ratio; PTT $=$ partial thromboplastin time; ALP = alkaline phosphatase; LF studies = lung function studies; ABG = arterial blood gases .

tive tests was not deficient and that alternative or additional tests would not have been helpful.

\section{Discussion}

Our data suggest that, in a tertiary referral centre, individualization of preoperative testing by a staff anesthesiologist is a more cost-effective approach to test selection relying on protocols or algorithms. The mean cost of preoperative investigations ordered in this manner was significantly lower than that incurred when test selection was driven by surgery-specific clinical pathway algorithms.

Preoperative assessment is thought to be a key process in minimizing the morbidity of surgery. Diagnostic studies should be used as an adjunct to the findings obtained from a review of the medical histo- ry and the subsequent physical examination or ordered to ensure that clinically silent conditions which can influence perioperative outcome are detected. In consequence, individualization of test selection should be the preferred approach to the use of diagnostic services in preoperative assessment. This is particularly relevant in the assessment of patients presenting for surgery with multiple co-existing disease states. The majority of our sample population was considered to have moderate or severe systemic disease by ASA classification reflecting the tertiary care status of the hospital where the study was conducted. Despite this, the mean number of tests ordered in this high risk population was similar to that previously reported in healthy patients. ${ }^{13}$ This finding may reflect the effectiveness of the joint clinical pathway develop- 
ment process in reducing preoperative testing or a discriminating attitude on the part of our surgical staff.

Nevertheless, the cost of tests ordered in both groups was substantial. CXR examinations were the most expensive test ordered in both phases of the study. The benefit of CXR examinations performed as part of a preoperative evaluation process has long been questioned. ${ }^{14}$ There is convincing evidence that CXR examination yields little information of benefit in reducing the risk of a surgical procedure, even if abnormalities are detected. ${ }^{2}$ While a significant reduction in CXR examinations did occur in Group 2, it is surprising, given the aforementioned evidence, that staff anesthesiologists still ordered a CXR on $20 \%$ of patients they assessed. While these data compare favourably with the outcome of an active process to implement practice guidelines for preoperative radiological investigations, ${ }^{15}$ they suggest that further reductions are possible and appropriate. ${ }^{16}$

ECG investigations were ordered in 60\% of cases in both phases of the study, reflecting the recommendation by the American College of Cardiology /American Heart Association that an ECG examination form part of the baseline preoperative assessment of patients at risk of having cardiac disease. ${ }^{17}$ The usefulness of the ECG in risk stratification has been questioned, particularly when the test is ordered based on a patient's age rather than for any specific indication. ${ }^{18}$ This view is controversial, as in the elderly, silent myocardial infarction is not an infrequent occurrence and the availability of baseline ECG findings is very helpful in the subsequent diagnosis and management of suspected adverse cardiac events. ${ }^{19}$

In Group 2, requests for hemoglobin assessments increased and those for complete blood counts decreased, and blood cross match requests were less frequently ordered in lieu of type and screens. As a consequence of this altered pattern of practice, the laboratory was able to provide test results within one hour rather than at the end of the clinic day, the latter being an unanticipated consequence of our study.

The overall number of tests ordered were not reduced in Group 2 relative to Group 1, however, the total cost of tests ordered was markedly reduced when testing was individualized. When Group 2 data were analyzed based on whether tests were ordered by resident or staff anesthesiologists, it became apparent that the test ordering practices of resident staff did not differ from Group 1 data. Indeed, the significant difference in cost between Groups 1 and 2 occurred only because of the reduction in number and cost of tests ordered by staff anesthesiologists. This finding suggests that our education of resident staff in the selec- tion of appropriate preoperative investigations was deficient. A similar pattern of excessive test ordering by resident staff has been reported in the critical care and emergency medicine areas. ${ }^{20,21}$ Fortunately, education appears to be very effective in altering this behaviour pattern. ${ }^{22}$

All of the study patients were scheduled for procedures requiring postoperative admission and thus were a select population, in that most elective surgery is outpatient based. ${ }^{11}$ This fact may have influenced test selection. The role of preoperative testing, particularly in healthy individuals or outpatients undergoing relatively non-invasive procedures, is questionable. ${ }^{13,23}$ Imasogie et al. ${ }^{24}$ recently demonstrated that considerable cost savings could be achieved, without adverse consequences, by discontinuing routine testing in patients scheduled for cataract surgery. These data support other studies where, in the absence of evidence on history and physical examination of undiagnosed disease, little if any information of consequence was added by preoperative screening. ${ }^{25}$

We acknowledge that the number of adverse events in Group 2 was statistically higher than in Group 1. However, the event rate, even in Group 2 was less than $4 \%$, which compares favourably with complication rates recorded in other high-risk populations. We are reassured that our blinded chart reviewer could not attribute any adverse outcome to a deficit in testing preoperatively. Our reliance on the judgement of an individual physician in this fashion raises the question of bias. It is clear that structured collection of outcome information prospectively, as a matter of routine, would be very valuable in assessing the consequences of procedural changes in hospital processes.

We have demonstrated that selection of preoperative tests by staff anesthesiologists resulted in less testing and less cost than surgery-specific critical pathways or resident-driven test ordering. Secondary and unexpected findings were the probable over-ordering of CXR investigations and the different test ordering pattern of staff and resident anesthesiologists. The latter findings are relatively simple issues to address by education initiatives.

\section{References}

1 Greer AE, Irwin MG. Implementation and evaluation of guidelines for preoperative testing in a tertiary hospital. Anaesth Intensive Care 2002; 30: 326-30.

2 Charpak Y, Blery C, Chastang C, Szatan M, Fourgeaux $B$. Prospective assessment of a protocol for selective ordering of preoperative chest x-rays. Can J Anaesth 1988; 35: 259-64.

3 Fowkes FG, Davies ER, Evans KT, et al. Multicentre 
trial of four strategies to reduce use of a radiological test. Lancet 1986; 1: 367-70.

4 Davies JM, Pagenkopf D, Todd K, Werry B, Finegan BA. Comparison of selection of preoperative laboratory tests: the computer vs the anaesthetist. Can J Anaesth 1994; 41: 1156-60.

5 National Collaborating Centre for Acute Care. Preoperative tests. The use of routine preoperative tests for elective surgery. National Institute for Clinical Excellence. cited 6/25/2003; accessed:11-30-2004; Available from URL, http://www.nice.org.uk/pdf/ cg3niceguidelinea4.pdf.

6 Markey DW, McGowan J, Hanks JB. The effect of clinical pathway implementation on total hospital costs for thyroidectomy and parathyroidectomy patients. Am Surg 2000; 66: 533-8.

7 Koch MO, Smith JA Jr, Hodge EM, Brandell RA. Prospective development of a cost-efficient program for radical retropubic prostatectomy. Urology 1994; 44: 311-8.

8 Narr BJ, Warner ME, Schroeder DR, Warner MA. Outcomes of patients with no laboratory assessment before anesthesia and a surgical procedure. Mayo Clin Proc 1997; 72: 505-9.

9 Finegan BA. Preadmission and outpatient consultation clinics (Editorial). Can J Anaesth 1992; 39: 1009-11.

10 Matthey P, Finucane BT, Finegan BA. The attitude of the general public towards preoperative assessment and risks associated with general anesthesia. Can J Anesth 2001; 48: 333-9.

11 Bond DM. Pre-anesthetic assessment clinics in Ontario. Can J Anesth 1999; 46: 382-7.

12 Hilditch WG, Asbury AJ, Crawford JM. Pre-operative screening: criteria for referring to anaesthetists. Anaesthesia 2003; 58: 117-24.

13 Turnbull JM, Buck C. The value of preoperative screening investigations in otherwise healthy individuals. Arch Intern Med 1987; 147: 1101-5.

14 Anonymous. Preoperative chest radiology. National study by the Royal College of Radiologists. Lancet 1979; 2: 83-6.

15 Barazzoni F, Grilli R, Amicosante AM, et al. Impact of end user involvement in implementing guidelines on routine pre-operative tests. Int J Qual Health Care 2002; 14: 321-7.

16 Brorsson B, Arvidsson $S$. The effect of dissemination of recommendations on use. Preoperative routines in Sweden, 1989-91. Int J Technol Assess Health Care 1997; 13: 547-52.

17 Wertheim WA. Perioperative risk. Review of two guidelines for assessing older adults. American College of Cardiology and American Heart Association. Geriatrics 2000; 55: 61-6.
18 Liu LL, Dzankic S, Leung JM. Preoperative electrocardiogram abnormalities do not predict postoperative cardiac complications in geriatric surgical patients. J Am Geriatr Soc 2002; 50: 1186-91.

19 Rich $M W$. The preoperative electrocardiogram: have we reached the end of an era? (Editorial). J Am Geriatr Soc 2002; 50: 1301-3.

20 Griffith CH III, Wilson JF, Desai NS, Rich EC. Does pediatric housestaff experience influence tests ordered for infants in the neonatal intensive care unit? Crit Care Med 1997; 25: 704-9.

21 Sucov A, Bazarian JJ, deLahunta EA, Spillane L. Test ordering guidelines can alter ordering patterns in an academic emergency department. J Emerg Med 1999; 17: 391-7.

22 Dowling PT, Alfonsi G, Brown MI, Culpepper L. An education program to reduce unnecessary laboratory tests by residents. Acad Med 1989; 64: 410-2.

23 Wattsman TA, Davies RS. The utility of preoperative laboratory testing in general surgery patients for outpatient procedures. Am Surg 1997; 63: 81-90.

24 Imasogie N, Wong DT, Luk K, Chung F. Elimination of routine testing in patients undergoing cataract surgery allows substantial savings in laboratory costs. A brief report. Can J Anesth 2003; 50: 246-8.

25 Golub R, Cantu R, Sorrento JJ, Stein HD. Efficacy of preadmission testing in ambulatory surgical patients. Am J Surg 1992; 163: 565-70. 